\title{
Tissue- and breed-specific expression of the chicken fat mass- and obesity-associated gene (FTO)
}

\author{
C. Song, W.T. Song, J.T. Shu, Z.Y. Tao, W.Q. Zhu, C. Di and H.F. Li \\ Key Laboratory of Poultry, Heredity, and Breeding, \\ Jiangsu Institute of Poultry Science, Yangzhou, China \\ Corresponding author: H.F. Li \\ E-mail: 1hfxf_002@aliyun.com
}

Genet. Mol. Res. 14 (3): 10500-10506 (2015)

Received January 9, 2015

Accepted May 26, 2015

Published September 8, 2015

DOI http://dx.doi.org/10.4238/2015.September.8.11

\begin{abstract}
The fat mass- and obesity-associated gene (FTO) is involved in energy metabolism, but little is known about the chicken FTO gene. The objective of the current study was to detect chicken FTO expression patterns in the hypothalamus, liver, and skeletal muscle during development, and analyze the effects of age and breed on FTO expression. Real-time quantitative polymerase chain reaction results revealed that chicken FTO mRNA was expressed in all of the tissues tested. Chicken FTO exhibited tissue- and breed-specific patterns in the recessive White Plymouth Rock chicken and the Qingyuan partridge chicken. The highest FTO expression level was in the hypothalami of 1-week-old chicks. FTO mRNA was expressed more in the breast muscles and livers of recessive White Plymouth Rock chickens than those of Qingyuan partridge chickens at 1 and 8 weeks of age. These results indicate that FTO probably plays a significant role in energy metabolism at 1 week old, when chicks have undergone metabolic adaptations from yolk dependence to the utilization of exogenous feed.
\end{abstract}

Key words: Chicken FTO; Hypothalamus; Liver; Skeletal muscle; Breed 


\section{INTRODUCTION}

The fat mass- and obesity-associated gene (FTO) has been found to be significantly associated with the human body mass index (Weedon et al., 2007), obesity, and type 2 diabetes by several independent genome-wide association studies (Grant et al., 2008; Attaoua et al., 2009; Li et al., 2010).

The FTO gene is highly conserved, and is found in vertebrates such as fish, chickens, and humans; this suggests that its ancestor was extant 450 million years ago (Fredriksson et al., 2008). Considerable progress has been made in understanding the function of FTO. FTO encodes a protein that is homologous to the DNA repair AlkB protein. The AlkB family proteins utilize iron, $\alpha$-ketoglutarate, and dioxygen to perform the oxidative repair of alkylated nucleobases in DNA and RNA (Jia et al., 2008). FTO loss in mice leads to postnatal growth retardation and a significant reduction in adipose tissue and lean body mass, but body composition remains relatively normal. Consistent with growth retardation, FTO mutant mice have reduced serum levels of IGF-1. FTO is functionally involved in energy homeostasis by the control of energy expenditure (Fischer et al., 2009). FTOdeficient mice (FtoI367F) are lean and protected from obesity (Church et al., 2009). In addition, FTO is involved in the regulation of food intake; the overexpression of FTO in mice leads to increased food intake and results in obesity (Church et al., 2010). The results of several studies suggest that food intake is greater in humans who carry at-risk FTO single nucleotide polymorphisms (Cecil et al., 2008; Speakman et al., 2008; Timpson et al., 2008; Wardle et al., 2009). Moreover, the specific deletion of FTO in the nervous system results in similar phenotypes as a whole-body deletion, indicating that FTO functions in the central nervous system to regulate postnatal growth (Gao et al., 2010). However, little is known about the avian FTO gene and its function.

The recessive White Plymouth Rock chicken has been intensively selected over many generations for meat production, and the Qingyuan partridge chicken is an important indigenous breed that is found in Qingyuan city, Guangdong Province, China. It is a light body-type breed with good meat quality. The two chicken breeds differ greatly in their growth efficiency and behavior. In the present study, FTO mRNA expression profiles in different tissues of the White Plymouth Rock and Qingyuan partridge chicken were detected by realtime polymerase chain reaction (PCR), and FTO tissue-specific expression in the two breeds at different ages was also analyzed.

\section{MATERIAL AND METHODS}

\section{Materials}

The eggs of recessive White Plymouth Rock and Qingyuan partridge chickens from the same incubation batch were selected at the National Chicken Genetic Resources, Jiangsu, China. The chickens were reared under standard conditions of light and temperature. The hypothalami, livers, breast muscles, and leg muscles were collected from embryos at 8,12 , 16 , and 20 days of age (ED8, etc.) and from chickens at 1, 8, 12, 14, and 16 weeks of age (W1, etc.) $(\mathrm{N}=5-7)$. The samples were immediately immersed in liquid nitrogen and stored at $-70^{\circ} \mathrm{C}$. 


\section{Main reagents and instruments}

TRNzol-A+ total RNA extraction reagent (DP421), SuperReal Premix (SYBR Green) (FP204-01), and QuantScript RT Kit (KR103-04) were obtained from Tiangen Biotech (Beijing) Co. Ltd. The instruments used were the PCR system 9700, the ABI 7500 Real-Time PCR System, a gel imaging system (Tanon 2500), and an Eppendorf BioPhotometer.

\section{Extraction of total RNA and selection of internal reference gene}

Total RNA was extracted following the instructions of the TRNzol-A+ total RNA extraction reagent, and was detected on $1.0 \%$ denaturing formaldehyde agarose gel. RNA concentration was measured using a BioPhotometer. Subsequently, $2 \mu \mathrm{g}$ total RNA was used as a template to synthesize the first-strand cDNA, following the instructions of the reverse transcription kit. ACTB was selected as the reference gene by conducting a quantitative fluorescence test.

\section{Design of primers and real-time PCR}

The target and reference genes were amplified by quantitative fluorescence PCR using the primers listed in Table 1.

\begin{tabular}{llr}
\multicolumn{2}{c}{ Table 1. Primers of target genes used in the real-time PCR. } \\
\hline Gene & Primer sequence (F: forward, R: reverse) & Product length (bp) \\
\hline ACTB & F: 5'-gtccaccttccagcagatgt-3' & 169 \\
FTO & R: 5'-ataaagccatgccaatctcg-3' & 159 \\
& F: 5'-ctggtcctccaagaagttcg-3' & \\
\hline
\end{tabular}

The conditions of the quantitative fluorescence PCR amplification system (total volume of $20 \mu \mathrm{L}$ ) were as follows: $10 \mu \mathrm{L} 2 \mathrm{X}$ SuperReal Premix, $1.5 \mu \mathrm{L}$ primers $(10 \mathrm{pmol} / \mu \mathrm{L}), 0.4 \mu \mathrm{L} 50 \mathrm{X}$ ROX reference dye, $2.5 \mu \mathrm{L}$ template (cDNA), and $\mathrm{ddH}_{2} \mathrm{O}$. The PCR started with predenaturing at $95^{\circ} \mathrm{C}$ for $15 \mathrm{~min}$, followed by 40 cycles of denaturing at $95^{\circ} \mathrm{C}$ for $15 \mathrm{~s}$, annealing at $55^{\circ} \mathrm{C}$ for $20 \mathrm{~s}$, and an extension at $72^{\circ} \mathrm{C}$ for $32 \mathrm{~s}$. The melting curve and electrophoretogram of the PCR products showed no other products except for those expected. The $2^{-\Delta \Delta C t}$ method was used to analyze the real-time PCR data relative to the average value of the control (Livak and Schmittgen 2001).

\section{Statistical analysis}

All of the statistical analyses were performed using SPSS 20.0 for Windows, and the data are reported as means \pm SE. Comparisons between groups were performed using one-way analysis of variance, with the significance level set at $\mathrm{P}<0.05$.

\section{RESULTS}

\section{Body weights of the two varieties}

There were significant differences in body weight at W1, W8, W12, and W14 (Table 2). 
Table 2. Body weight of Recessive White Rock chicken and Qingyuan partridge chicken.

\begin{tabular}{llrll}
\hline Body weight & W1 & \multicolumn{1}{c}{ W8 } & W12 & W14 \\
\hline Qingyuan partridge chicken & $56.22 \pm 0.592^{*}$ & $584.04 \pm 9.84^{*}$ & $1009.96 \pm 18.03^{*}$ & $1173.47 \pm 18.68^{*}$ \\
Recessive White Rock chicken & $84.20 \pm 0.93$ & $1093.44 \pm 15.65$ & $1962.14 \pm 28.87$ & $2249.35 \pm 33.20$ \\
\hline
\end{tabular}

*Significant difference between the two breeds in the same age $(\mathrm{P}<0.05)$.

\section{FTO mRNA expression in different tissues of the two varieties}

During embryonic development in the hypothalamus, FTO mRNA levels at ED8 and ED12 were higher than at ED16 and ED20. At ED16, FTO mRNA levels in the Qingyuan partridge chicken were higher than those in the recessive White Plymouth Rock chicken; at W14, FTO mRNA levels in the recessive White Plymouth Rock chicken were higher than those in the Qingyuan partridge chicken. The expression of FTO mRNA at W1 was significantly higher than at other ages $(\mathrm{P}<0.01$; Figure 1$)$.

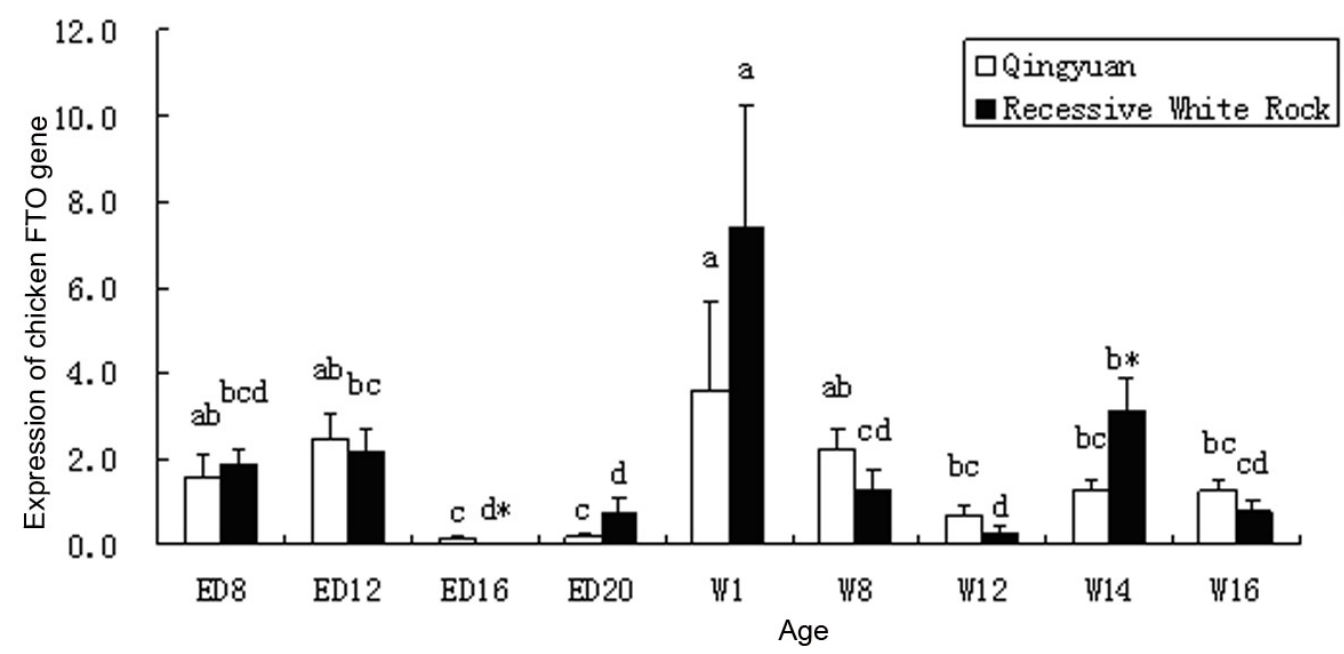

Figure 1. FTO mRNA expression in hypothalamus of Recessive White Rock chicken and Qingyuan partridge chicken. Values with the same letter are not significantly different between different embryo age in the same breed $(\mathrm{P}>0.05)$, values with the different letters are significantly different between different ages in the same breed $(\mathrm{P}<$ $0.05)$. *Significant difference between the two breeds in the same age $(\mathrm{P}<0.05)$.

In the recessive White Plymouth Rock chicken, FTO mRNA levels in the liver at W1 were higher than at other ages, but a similar pattern was not found in the Qingyuan partridge chicken (Figure 2). In the recessive White Plymouth Rock chicken, the highest levels of FTO mRNA expression were detected at ED20, W1, and W8, when they were significantly higher than those in the Qingyuan partridge chicken (Figure 3). In the leg muscle, FTO mRNA levels in the recessive White Plymouth Rock chicken at W8 were higher than those in the Qingyuan partridge chicken (Figure 4). 


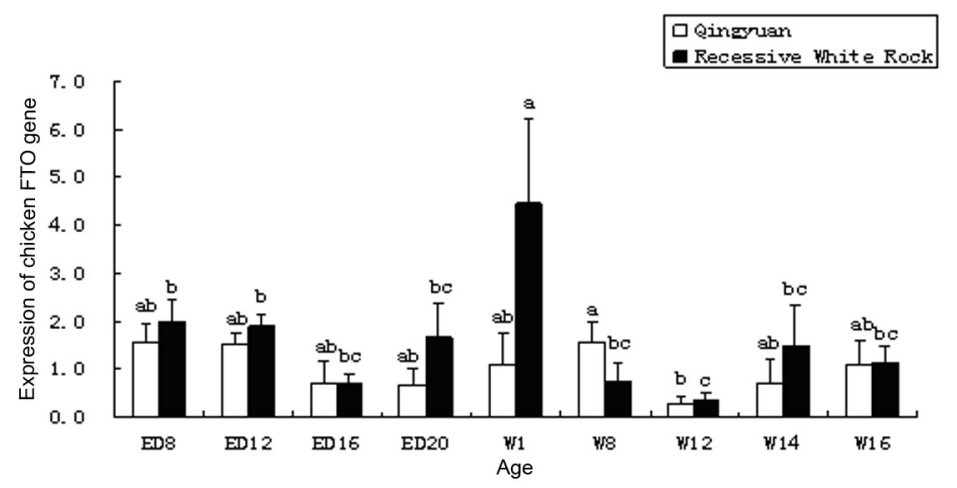

Figure 2. FTO mRNA expression in liver of Recessive White Rock chicken and Qingyuan partridge chicken. Values with the same letter are not significantly different between different embryo age in the same breed $(\mathrm{P}>$ $0.05)$, values with the different letters are significantly different between different ages in the same breed $(\mathrm{P}<0.05)$.

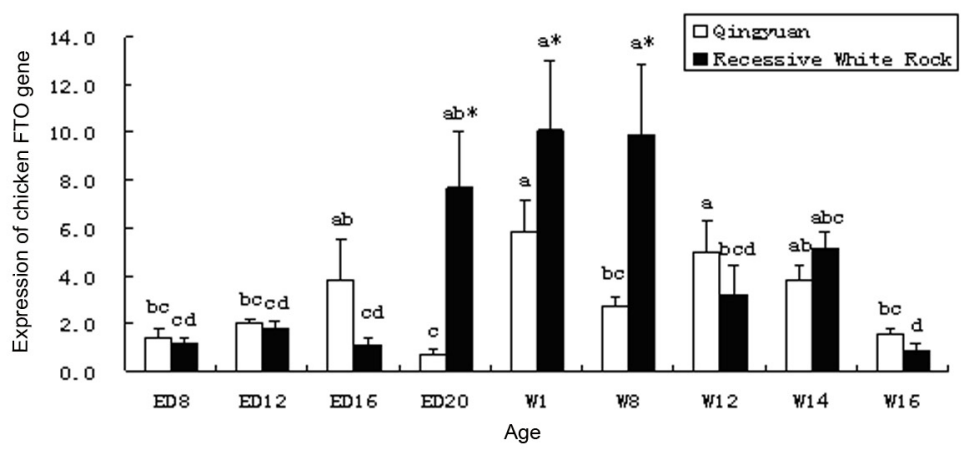

Figure 3. FTO mRNA expression in breast muscle of Recessive White Rock chicken and Qingyuan partridge chicken. Values with the same letter are not significantly different between different embryo age in the same breed $(\mathrm{P}>0.05)$, values with the different letters are significantly different between different ages in the same breed $(\mathrm{P}<$ 0.05). *Significant difference between the two breeds in the same age $(\mathrm{P}<0.05)$.

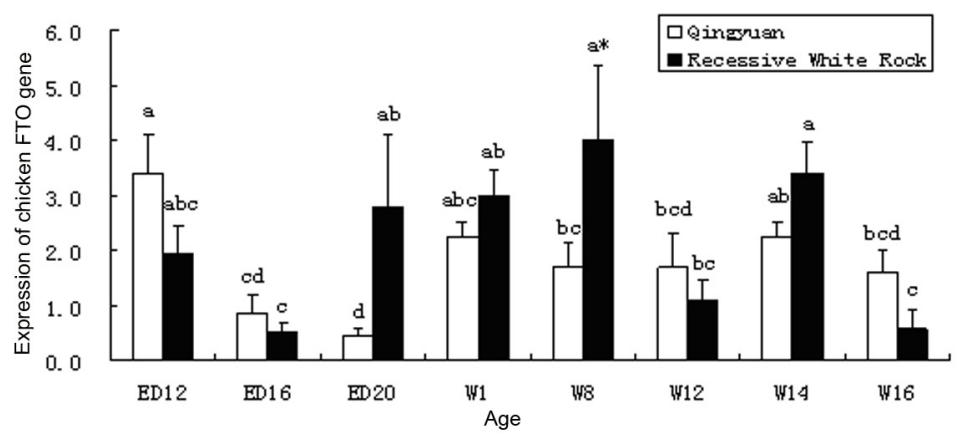

Figure 4. FTO mRNA expression in leg muscle of Recessive White Rock chicken and Qingyuan partridge chicken. Values with the same letter are not significantly different between different embryo age in the same breed $(\mathrm{P}>$ $0.05)$, values with the different letters are significantly different between different ages in the same breed $(\mathrm{P}<0.05)$. *Significant difference between the two breeds in the same age $(\mathrm{P}<0.05)$. 


\section{DISCUSSION}

According to the real-time quantitative fluorescence PCR results, chicken FTO was expressed in all of the tissues tested. However, the expression patterns of the two breeds were different: in the recessive White Plymouth Rock, the highest FTO expression was detected in the breast muscle at ED20, W1, and W8, while in the Qingyuan partridge chicken the highest FTO expression in the breast muscle was detected at $\mathrm{W} 1$ and $\mathrm{W} 12$. When comparing different ages, the highest FTO mRNA level was found at W8 in the recessive White Plymouth Rock chicken in the leg muscle; this result is similar to that found in a previous study, which found high FTO mRNA levels in the skeletal muscle of 8-week-old Cobb chickens (Tiwari et al., 2012).

There are striking phenotypic differences between the Qingyuan partridge chicken and the recessive White Plymouth Rock chicken. The two breeds provide excellent models for studying the mechanisms that regulate body size and composition. In the current study, high FTO mRNA levels were found in the breast muscle of recessive White Plymouth Rock chickens, but not in the hypothalamus at W8; this result differs to those of a previous study in layers and broilers of different ages (Wang et al., 2012). The increase in FTO mRNA levels in the breast muscle may be due to the rapid growth of skeletal muscle and body weight in commercial broilers, which have been selectively bred for body weight gain, enlarged breast muscles, and food intake (Tiwari et al., 2012). This pattern was not found in the breast muscle of Qingyuan partridge chickens because they have not been selectively bred; the highest expression in the breast muscle of this breed was detected at W1, W12, and W14, possibly because of the slow increase in body weight exhibited by this breed.

In mammals, FTO expression has been detected in hypothalamus adipose tissue and the liver (Fredriksson et al., 2008; Stratigopoulos et al., 2008; Madsen et al., 2010). In the rat, fasting reduces levels of FTO mRNA in the arcuate nucleus of the hypothalamus, a site where FTO expression is particularly high (Tung et al., 2010). These results suggest that FTO is involved in food intake regulation. In the present study, FTO expression was detected in the hypothalamus, liver, and skeletal muscle of the two breeds at different developmental stages. These results are consistent with those obtained in studies of layers and broilers (Yuan et al., 2009; Wang et al., 2012; Ibba et al., 2013).

The hypothalamus is important in energy balance, and the liver is also an important metabolism-related organ. In this study, FTO mRNA levels exhibited a similar pattern: after ED12, mRNA levels decreased; this may be due to chicken embryonic development, because at ED12 albumen absorption occurs. FTO mRNA levels increased to a peak at W1, and chickens at this age would have undergone metabolic adaptations from yolk dependence to the utilization of exogenous feed, and have recovered from the stress of hatching (Yuan et al., 2009).

In this study, the chicken FTO gene was expressed in several different tissues in addition to the hypothalamus, suggesting that FTO may be involved in energy metabolism in chicken skeletal muscle and liver. The present study also provides evidence that FTO is related to energy homeostasis, may play a role in breed-specific body weight, and genetic selection for meat production over many generations modifies FTO expression. Further studies are necessary to elucidate the role of chicken FTO in peripheral tissues, such as skeletal muscle, in energy metabolism.

\section{Conflicts of interest}

The authors declare no conflict of interest. 


\section{ACKNOWLEDGMENTS}

Research supported by the National Natural Science Foundation of China (\#31301967), the Jiangsu Science Youth Foundation (\#BK20140498), the Modern Agriculture Poultry Industry Technology System (\#CARS-42-GO3), and the National High Technology Research and Development Program (\#2011AA100301).

\section{REFERENCES}

Attaoua R, Ait El Mkadem S, Lautier C, Kaouache S, et al. (2009). Association of the FTO gene with obesity and the metabolic syndrome is independent of the IRS-2 gene in the female population of Southern France. Diabetes Metab. 35: 476-483.

Cecil JE, Tavendale R, Watt P, Hetherington MM, et al. (2008). An obesity-associated FTO gene variant and increased energy intake in children. New Engl. J. Med. 359: 2558-2566.

Church C, Lee S, Bagg EA, McTaggart JS, et al. (2009). A mouse model for the metabolic effects of the human fat mass and obesity associated FTO gene. PLoS Genet. 5: e1000599.

Church C, Moir L, McMurray F, Girard C, et al. (2010). Overexpression of Fto leads to increased food intake and results in obesity. Nat. Genet. 42: 1086-1092.

Fischer J, Koch L, Emmerling C, Vierkotten J, et al. (2009). Inactivation of the Fto gene protects from obesity. Nature 458: 894-898.

Fredriksson R, Hagglund M, Olszewski PK, Stephansson O, et al. (2008). The obesity gene, FTO, is of ancient origin, up-regulated during food deprivation and expressed in neurons of feeding-related nuclei of the brain. Endocrinology 149: 2062-2071.

Gao X, Shin YH, Li M, Wang F, et al. (2010). The fat mass and obesity associated gene FTO functions in the brain to regulate postnatal growth in mice. PLoS One 5: e14005.

Grant SF, Li M, Bradfield JP, Kim CE, et al. (2008). Association analysis of the FTO gene with obesity in children of Caucasian and African ancestry reveals a common tagging SNP. PLoS One 3: e1746.

Ibba A, Pilia S, Zavattari P, Loche A, et al. (2013). The role of FTO genotype on eating behavior in obese Sardinian children and adolescents. J. Pediatr. Endocrinol. Metab. 26: 539-544.

Jia G, Yang CG, Yang S, Jian X, et al. (2008). Oxidative demethylation of 3-methylthymine and 3-methyluracil in singlestranded DNA and RNA by mouse and human FTO. FEBS Lett. 582: 3313-3319.

Li M, Liu Y, Xu P, Ye M, et al. (2010). Association of the rs9939609 polymorphism of FTO gene with overweight or obesity in Hazakh children. Zhonghua Yi Xue Yi Chuan Xue Za Zhi 27: 678-681.

Livak KJ and Schmittgen TD (2001). Analysis of relative gene expression data using real-time quantitative PCR and the 2(-Delta Delta C(T)) method. Methods 25: 402-408.

Madsen MB, Birck MM, Fredholm M and Cirera S (2010). Expression studies of the obesity candidate gene FTO in pig. Anim. Biotechnol. 21: 51-63.

Speakman JR, Rance KA and Johnstone AM (2008). Polymorphisms of the FTO gene are associated with variation in energy intake, but not energy expenditure. Obesity 16: 1961-1965.

Stratigopoulos G, Padilla SL, Leduc CA, Watson E, et al. (2008). Regulation of Fto/Ftm gene expression in mice and humans. Am. J. Physiol. Regul. Integr. Comp. Physiol. 294: R1185-R1196.

Timpson NJ, Emmett PM, Frayling TM, Rogers I, et al. (2008). The fat mass- and obesity-associated locus and dietary intake in children. Am. J. Clin. Nutr. 88: 971-978.

Tiwari A, Krzysik-Walker SM and Ramachandran R (2012). Cloning and characterization of chicken fat mass and obesity associated (Fto) gene: fasting affects Fto expression. Domest. Anim. Endocrinol. 42: 1-10.

Tung YC, Ayuso E, Shan X, Bosch F, et al. (2010). Hypothalamic-specific manipulation of Fto, the ortholog of the human obesity gene FTO, affects food intake in rats. PLoS One 5: e8771.

Wang Y, Rao K, Yuan L, Everaert N, et al. (2012). Chicken FTO gene: tissue-specific expression, brain distribution, breed difference and effect of fasting. Comp. Biochem. Physiol. A Mol. Integr. Physiol. 163: 246-252.

Wardle J, Llewellyn C, Sanderson S and Plomin R (2009). The FTO gene and measured food intake in children. Int. J. Obes. 33: 42-45.

Weedon MN, Lettre G, Freathy RM, Lindgren CM, et al. (2007). A common variant of HMGA2 is associated with adult and childhood height in the general population. Nat. Genet. 39: 1245-1250.

Yuan L, Ni Y, Barth S, Wang Y, et al. (2009). Layer and broiler chicks exhibit similar hypothalamic expression of orexigenic neuropeptides but distinct expression of genes related to energy homeostasis and obesity. Brain Res. 1273: 18-28. 